\title{
Dengue: an update for clinicians working in non-endemic areas
}

\author{
Authors: Sophie Yacoub ${ }^{A}$ and Bridget Wills ${ }^{B}$
}

Dengue is one of the most rapidly emerging viral infections globally, with 2.5 billion people now thought to live in dengue-endemic areas. In addition, reports of travel-related and autochthonous infections are increasing in non-endemic areas. Most patients with dengue experience a selflimiting febrile illness, but a proportion develop potentially life threatening complications around the time of fever clearance, including plasma leakage occasionally leading to shock, bleeding, and organ impairment. As dengue can present with non-specific symptoms of fever, headache and myalgias, the potential for misdiagnosis and inappropriate management by medical staff inexperienced with the disease is a concern. This short review will outline the latest World Health Organisation disease classification, potential complications, clinical assessment and management for clinicians working in non-endemic areas.

Dengue is the most abundant vector-borne viral disease in the world. In the last 20 years the burden of disease has increased four-fold and 2.5 billion people are now thought to live in dengue-endemic areas. Latest estimates suggest there are around 100 million symptomatic dengue infections annually. ${ }^{1}$ South-East Asia has a particularly high disease burden, although in the last two decades Latin America and the Caribbean have also seen considerable expansion in case numbers. More recently, outbreaks have been reported from several African countries. ${ }^{2,3}$

Dengue can be caused by any one of four viral serotypes (dengue virus (DENV) 1-4), and is transmitted by day-biting urban-adapted Aedes mosquito species. ${ }^{4}$ After an incubation period of 4-14 days, symptomatic patients typically experience

Authors: ${ }^{A}$ clinical research fellow, Oxford University Clinical Research Unit, Wellcome Trust Major Overseas Programme, Hospital for Tropical Diseases, Ho Chi Minh City, Vietnam, and specialist registrar in infectious diseases, Imperial College, Department of Medicine, London, UK; ${ }^{B}$ head of the dengue group at the Oxford University Clinical Research Unit, Wellcome Trust Major Overseas Programme, Hospital for Tropical Diseases, Ho Chi Minh City, Vietnam, and professor of tropical medicine, Nuffield Department of Clinical Medicine, Centre for Tropical Medicine, Oxford, UK a self-limiting febrile illness with one or more of the following symptoms:

$>$ high fever of up to $40^{\circ} \mathrm{C}$

$>$ headache

$>$ retro-orbital pain

$>$ nausea/vomiting

$>$ myalgia

$>$ arthralgia

$>$ rashes (Fig 1).

This febrile phase lasts for 4-7 days, and once the temperature settles most patients recover without complications. ${ }^{5}$ Crucially however, a small number of individuals go on to develop potentially serious complications during the 48 hours around defervescence (the critical phase) including plasma leakage, sometimes severe enough to cause hypovolaemic shock; coagulation derangements, sometimes associated with haemorrhage; and organ impairment (usually secondary to severe leakage and/or bleeding) ${ }^{6}$ With careful management the outcome is generally good and convalescence is typically short and uneventful, although it may be prolonged in adults with profound tiredness, aesthenia and depression persisting for weeks to months after recovery. ${ }^{7}$

The World Health Organisation (WHO) classification of dengue was updated in $2009,{ }^{6}$ replacing the original dengue fever/dengue haemorrhagic fever grading, and now comprises the following entities:

$>$ dengue

$>$ dengue with warning signs (Box 1)

$>$ severe dengue (Box 2).

\section{Box 1. Warning signs.}
Abdominal pain or tenderness
Persistent vomiting
Clinical fluid accumulation
Mucosal bleeding
Lethargy/restlessness
$>$ Liver enlargement $>2 \mathrm{~cm}$
$>$ Increase in hematocrit concurrent with rapid decrease in platelet count 
(a)

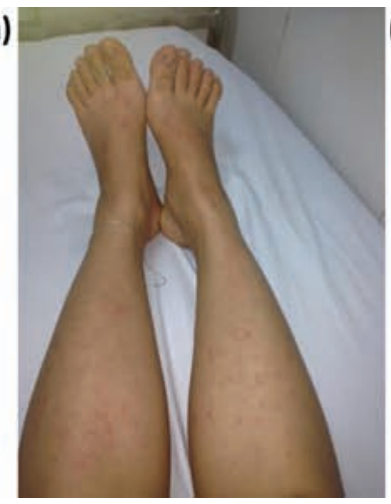

(b)

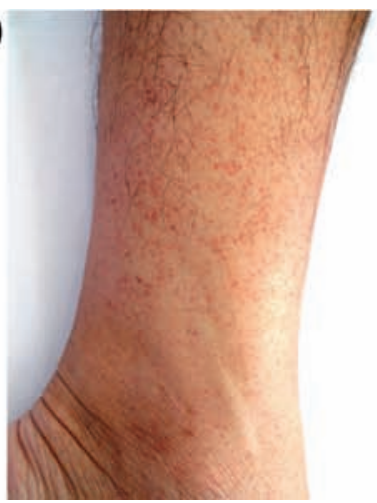

(c)

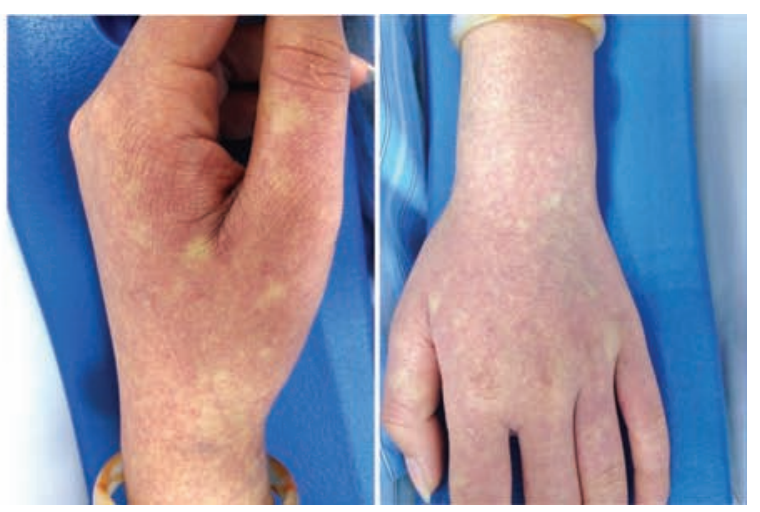

Fig 1. Spectrum of dengue associated rashes. (a) Macular-papular rash may be seen during the febrile phase; (b) petechial rash may develop during the febrile/critical phase particularly on arms and legs; (c) erythematous rash with 'islands of white' can be widespread and develop during the recovery phase.

Development of severe dengue is strongly associated with secondary heterotypic DENV infections rather than primary infections. ${ }^{8}$ Age also has a strong influence on the clinical phenotype, with severe plasma leakage more likely to occur in children, while major bleeding is usually seen in adults. ${ }^{9}$

\section{Dengue in non-endemic areas}

Changing mosquito ecology, globalisation of trade and a remarkable increase in the volume of air travel have all influenced dengue epidemiology. Reports of travellers acquiring dengue are increasing, as are reports of autochthonous cases presenting in non-endemic areas where the mosquito vectors have become newly established. ${ }^{10}$ Surveillance data indicate that dengue is now the most common cause for fever in travellers returning from all geographical regions except sub-Saharan Africa and Central America, where malaria remains the most common cause. ${ }^{11,12}$ Travellers returning to Europe usually acquire dengue from South-East Asia, particularly Thailand, ${ }^{13}$ while in the USA the highest rates followed travel to Dominican Republic and Mexico. ${ }^{14}$ Autochthonous spread has been reported from the south of France and Croatia, and in 2012 the first European dengue outbreak since the 1920's occurred in Madeira, resulting in over 2,000 cases and 120 hospitalisations. ${ }^{15}$

\section{Box 2. Criteria for severe dengue.}

$$
\begin{aligned}
& \text { > Severe plasma leakage leading to: } \\
& \text { - shock } \\
& \text { - fluid accumulation with respiratory distress. } \\
& \text { > Severe bleeding } \\
& \text { > Severe organ involvement: } \\
& \text { - liver: AST or ALT } \geq 1,000 \\
& \text { - CNS: impaired consciousness } \\
& \text { - heart and other organs. }
\end{aligned}
$$

$\mathrm{ALT}=$ alanine aminotransferase; $\mathrm{AST}$ = aspartate aminotransferase; $\mathrm{CNS}=$ central nervous system.
Although most patients with travel-associated dengue have primary infections and thus are unlikely to develop severe disease, one study from Europe showed $11 \%$ of the 219 dengue infections reported between 2003-5 had complications, including internal haemorrhage, plasma leakage and shock. ${ }^{16}$ Recent reports of fatalities in Germany and Norway have highlighted the potential for misdiagnosis and inappropriate management by staff unfamiliar with the disease. ${ }^{17,18}$

\section{Clinical assessment and laboratory diagnosis}

In the early febrile phase dengue presents with clinical features similar to malaria and a wide variety of bacterial and viral infections. ${ }^{6}$ A detailed travel history is important, focused on timing and duration of stay in endemic areas, dengue seasonality and epidemic activity in the places visited, and predominant urban or rural stay. ${ }^{19}$ Simple laboratory investigations may help distinguish dengue from other infections, but are not definitive. Thrombocytopenia occurs almost universally, with a nadir around defervescence, and can help differentiate dengue from infections like influenza, measles and rubella. Leucopenia and a mild transaminitis are also seen, but other laboratory parameters are generally unaffected unless complications develop. Table 1 briefly summarises the clinical features and investigations to consider during each disease phase.

In the first week of illness, laboratory confirmation relies on identification of viral RNA using reverse transcription polymerase chain reaction (RT-PCR), or detection of dengue non-structural protein 1 (NS1) using enzyme-linked immunosorbent assay (ELISA). ${ }^{22,23}$ However, the viraemia is short so serodiagnostic tests (typically IgM Capture ELISA) are used from the end of the first week onwards, ideally with demonstration of seroconversion on paired samples. Rapid tests, often combining NS1 detection with an antibody test, are also available and have a reasonable window of detection. ${ }^{24}$

\section{Management}

A number of warning signs (Box 1) may occur during the transition into the critical phase. Of note, abdominal pain can be severe, and there are reports of surgery being performed for suspected acute abdomen before dengue was diagnosed..$^{17,25}$ Symptomatic relief and regular outpatient review are usually 
Table 1. Assessment of suspected dengue patients during each disease phase.

\section{Febrile phase}

Clinical

$>$ Focus on dengue warning signs.

$>$ Assess for signs of other infections suggested by travel history/ epidemiological links (eg an eschar in rickettsial infections, prominent arthralgia in chikungunya, Koplik spots in measles, etc).

Haematology

> Baseline haemoglobin/haematocrit to facilitate later assessment of haemoconcentration/plasma leakage.

$>$ Early leucopenia and thrombocytopenia support dengue diagnosis

> Coagulation usually within normal range, baseline values are useful later.

Biochemistry

> Liver enzymes and creatine kinase may be elevated.

$>$ Renal function usually normal. May be deranged with persistent vomiting or comorbidities.

Imaging

> Ultrasound to assess early signs of plasma leakage, particularly gall bladder wall thickening. ${ }^{20}$

\section{Critical phase}

Clinical

> Careful haemodynamic assessment. Cool, clammy peripheries, tachycardia and pulse pressure narrowing usually precede hypotensive shock from plasma leakage.

$>$ Watch for fluid accumulation, especially pleural effusions and ascites.

> Many patients have no clinical bleeding. Mucosal (often gastrointestinal) bleeding usually follows prolonged/profound shock, but may occur without shock especially in adults.

Haematology

> Rising haemoglobin/haematocrit suggests significant plasma leakage and risk of progression to severe dengue.

> Typical coagulopathy shows prolonged APTT with low fibrinogen, but PT usually normal.

> Severity of thrombocytopenia and coagulopathy reflect the severity of plasma leakage.

Biochemistry

> Liver enzymes tend to peak during this phase.

$>$ Renal function usually preserved unless prolonged hypotensive shock.

Imaging

> Ultrasound to assess serosal fluid accumulation.

$>$ Echocardiography to assess cardiac function in fluid refractory shock or if myocarditis suspected. ${ }^{21}$

\section{Recovery phase}

Clinical

> If intravenous fluids are continued into this phase, patients are at risk of fluid overload.

Haematology

$>$ Most parameters normalise.

> Haemoglobin/haemtocrit may fall temporarily below baseline as extravasated fluid is reabsorbed.

Biochemistry

> Liver enzymes gradually normalise but often lag behind other parameters.

Other investigations

$>$ Bradyarrythmias can be observed but are usually benign. ECG should be performed if symptomatic.

APTT $=$ activated partial thromboplastin time; $\mathrm{PT}=$ prothrombin time

\section{Key points}

Dengue is emerging as the most abundant vector-borne viral illness in the world.

Travel-related and autochthonous infections are increasing in non-endemic areas.

Dengue can present with non-specific symptoms of fever, headache and myalgias and the potential for misdiagnosis and inappropriate management by medical staff unfamiliar with the disease is a cause for concern.

Most patients with dengue experience a self-limiting febrile illness but a proportion develop potentially life-threatening complications around the time of fever clearance, including plasma leakage occasionally leading to shock, bleeding and organ impairment.

Management currently relies on symptomatic and supportive treatment in the form of meticulous fluid balance for those identified to have significant plasma leakage.

KEYWORDS: Dengue, update, travel, clinical, management

sufficient in the absence of warning signs. Other high-risk groups include children and the elderly, pregnant women, and individuals with comorbidities (eg diabetes, hypertension or obesity). ${ }^{26,27}$ These patients should be seen at least once daily or admitted for close observation.

No specific antivirals or adjunctive therapies have shown benefit, so management relies on symptomatic and supportive treatment. ${ }^{28,29}$ This involves meticulous fluid balance and cautious intravenous fluid replacement for patients unable to tolerate oral fluids and those identified to have significant plasma leakage. The minimum volume of parenteral fluid should be given to achieve adequate organ perfusion while avoiding the well-recognised complication of fluid overload. Oral paracetamol can be given but NSAIDs should be avoided due to the risk of gastrointestinal bleeding. Specific management for patients who develop severe dengue is out of the scope of this article and the WHO guidelines should be consulted. ${ }^{6}$

\section{Conclusion}

Dengue is one of the most rapidly emerging viral infections in the world, and as it continues to spread across the globe, travel-related infections are likely to increase. As climate change and globalisation facilitate the establishment of competent vectors in non-endemic areas, local transmission could also become more frequent. Awareness of dengue, the potential complications, and the essential principles of management is important for frontline healthcare personnel everywhere to avoid unnecessary morbidity and mortality.

\section{References}

1 Bhatt S, Gething PW, Brady OJ et al. The global distribution and burden of dengue. Nature 2013;496:504-7. 
2 Sessions OM, Khan K, Hou Y et al. Exploring the origin and potential for spread of the 2013 dengue outbreak in Luanda, Angola. Glob Health Action 2013;6:21822.

3 Amarasinghe A, Kuritsk JN, Letson GW, Margolis HS. Dengue virus infection in Africa. Emerg Infect Dis 2011;17:1349-54.

4 Yacoub S, Farrar J. Dengue. In: Manson's Tropical Diseases, 23rd edn. Philadelphia: Saunders Ltd, 2013: 162-70.

5 Whitehorn J, Farrar J. Dengue. Clin Med 2011;11:483-7.

6 World Health Organization. Dengue: Guidelines for treatment, prevention and control. Geneva: WHO, 2009.

7 Seet RC, Quek AM, Lim EC. Post-infectious fatigue syndrome in dengue infection. J Clin Virol 2007;38:1-6.

8 Guzman MG, Alvarez M, Halstead SB. Secondary infection as a risk factor for dengue hemorrhagic fever/dengue shock syndrome: an historical perspective and role of antibody-dependent enhancement of infection. Arch Virol 2013;158:1445-59.

9 Trung DT, Thao le TT, Dung NM et al. Clinical features of dengue in a large Vietnamese cohort: intrinsically lower platelet counts and greater risk for bleeding in adults than children. PLoS Negl Trop Dis 2012;6:e1679.

10 Odolini S, Parola P, Gkrania-Klotsas E et al. Travel-related imported infections in Europe, EuroTravNet 2009. Clin Microbiol Infect 2012;18:468-74.

11 Freedman DO, Weld LH, Kozarsky PE et al. Spectrum of disease and relation to place of exposure among ill returned travelers. N Engl J Med 2006;354:119-30.

12 Field V, Gautret P, Schlagenhauf $\mathrm{P}$ et al. Travel and migration associated infectious diseases morbidity in Europe, 2008. BMC Infect Dis 2010;10:330.

13 Rocklov J, Lohr W, Hjertqvist M, Wilder-Smith A. Attack rates of dengue fever in Swedish travellers. Scand J Infect Dis 2014;46:412-7.

14 Centers for Disease Control and Prevention (CDC). Travelassociated Dengue surveillance - United States, 2006-2008. MMWR Morb Mortal Wkly Rep 2010;59:715-9.

15 Tomasello D, Schlagenhauf P. Chikungunya and dengue autochthonous cases in Europe, 2007-2012. Travel Med Infect Dis 2013;11:274-84.

16 Wichmann O, Gascon J, Schunk M et al. Severe dengue virus infection in travelers: risk factors and laboratory indicators. J Infect Dis 2007;195:1089-96.

17 Schmidt-Chanasit J, Tenner-Racz K, Poppert D et al. Fatal dengue hemorrhagic fever imported into Germany. Infection 2012;40:441-3.
18 Vainio K, Noraas S, Holmberg M et al. Fatal and mild primary dengue virus infections imported to Norway from Africa and south-east Asia, 2008-2010. Euro Surveill 2010;15:pii=19666.

19 Wilder-Smith A, Schwartz E. Dengue in travelers. N Engl J Med 2005;353:924-32.

20 Michels M, Sumardi U, de Mast Q et al. The predictive diagnostic value of serial daily bedside ultrasonography for severe dengue in Indonesian adults. PLoS Negl Trop Dis 2013;7:e2277.

21 Yacoub S, Wertheim H, Simmons CP, Screaton G, Wills B. Cardiovascular manifestations of the emerging dengue pandemic. Nat Rev Cardiol 2014;11:335-45.

22 Hang VT, Nguyet NM, Trung DT et al. Diagnostic accuracy of NS1 ELISA and lateral flow rapid tests for dengue sensitivity, specificity and relationship to viraemia and antibody responses. PLoS Negl Trop Dis 2009;3:e360.

23 Tang KF, Ooi EE. Diagnosis of dengue: an update. Expert Rev Anti Infect Ther 2012;10:895-907.

24 Fry SR, Meyer M, Semple MG et al. The diagnostic sensitivity of dengue rapid test assays is significantly enhanced by using a combined antigen and antibody testing approach. PLoS Negl Trop Dis 2011;5:e1199.

25 McFarlane ME, Plummer JM, Leake PA et al. Dengue fever mimicking acute appendicitis: A case report. Int J Surg Case Rep 2013;4:1032-4.

26 Anders KL, Nguyet NM, Chau NV et al. Epidemiological factors associated with dengue shock syndrome and mortality in hospitalized dengue patients in Ho Chi Minh City, Vietnam. Am J Trop Med Hyg 2011;84:127-34.

27 Pang J, Salim A, Lee VJ et al. Diabetes with hypertension as risk factors for adult dengue hemorrhagic fever in a predominantly dengue serotype 2 epidemic: a case control study. PLoS Negl Trop Dis 2012;6:e1641.

28 Low JG, Sung C, Wijaya L et al. Efficacy and safety of celgosivir in patients with dengue fever (CELADEN): a phase $1 \mathrm{~b}$, randomised, double-blind, placebo-controlled, proof-of-concept trial. Lancet Infect Dis 2014;14:706-15.

29 Tam DT, Ngoc TV, Tien NT et al. Effects of short-course oral corticosteroid therapy in early dengue infection in Vietnamese patients: a randomized, placebo-controlled trial. Clin Infect Dis 2012;55:1216-24.

Address for correspondence: Dr S Yacoub, Oxford University Clinical Research Unit, Wellcome Trust Major Overseas Programme, Hospital for Tropical Diseases, 764 Vo Van Kiet street, Ho Chi Minh City, Vietnam.

Email: syacoub@imperial.ac.uk 